\title{
El entramado de actores entre política social y economía social en Argentina: una mirada relacional local
}

\author{
The structure of actors between social policy \\ and social economy in Argentina: a relational look local
}

Cynthia Ferrari Mango*

\begin{abstract}
Resumen
El Ministerio de Desarrollo Social de la Nación de Argentina, durante el segundo gobierno de Cristina Fernández de Kirchner (2011-2015), promulgó en sus políticas sociales una orientación territorial y principios de la economía social y solidaria. A su vez, fomentó la participación de gobiernos subnacionales y universidades públicas en las diferentes etapas de la política pública. Dichos lineamientos, se plasmaron en el Programa Ingreso Social con Trabajo Argentina Trabaja (Prist - At). En este marco, nos proponemos caracterizar la dimensión relacional de la política social analizando el entramado de actores que conforma el Prist - At y las relaciones entre ellos en el espacio local de gestión. La metodología utilizada es cualitativa e incluye diversas técnicas de recolección de información como entrevistas, observación y análisis de documentos oficiales.
\end{abstract}

Palabras clave: entramado de actores, espacio local de gestión, política social, programa social ingreso con trabajo - argentina trabaja.

\section{Abstract}

The Ministry of Social Development of the Nation of Argentina, during the second government of Cristina Fernández de Kirchner (2009-2015), promulgated in its social policies a territorial orientation and principles of the social and solidarity economy. At the same time, it encouraged the participation of governments and universities in the different stages of public policy. These guidelines were embodied in the Social Income with Work Program - Argentina Trabaja (Prist At). In this framework, we propose to characterize the relational dimension of social policy by analyzing the network of actors that make up the principle - In the relations between them in the local management space. The methodology has been classified qualitatively and includes information gathering techniques such as interviews, observation and analysis of official documents.

Keywords: framework of actors, local management space, social policy, social program income with work - argentina works.

\section{(CC) $($ (1) $\circledast$ (2)}

Recibido: I De JUlio de 2019 | Aprobado: 2 De OCtubre De 2019

\section{Cómo CITAR ESTE ARTICULO}

Ferrari Mango, C. (2020). El entramado de actores entre política social y economía social en Argentina: Una mirada relacional local. Collectivus, Revista de Ciencias Sociales, 7(1), 121-136. DOI: https://doi.org/10.15648/Collectivus.vol7num1.2020.2556

*Doctora en Ciencias Sociales. Universidad de Buenos Aires (UBA). Magíster en Políticas Públicas por la Facultad Latinoamericana de Ciencias Sociales (FLACSO) - Sede académica Argentina. FLACSO Tucumán 1966, piso 2, C1050AAN Caba. Buenos Aires, Argentina. cferrari@flacso.org.ar / ferrarimangoc@gmail.com 


\section{Introducción}

La política social es el vehículo que nos permite comprender la interacción entre los actores sociales y el Estado, en tanto instancia de articulación de relaciones sociales (Isuani, 2008; Oszlak, 1997). A su vez, el campo de las mimas está atravesado por luchas y enfrentamientos de diferentes fuerzas. Los actores no se desenvuelven en el vacío, sino que están determinados por un marco de institucionalidad en el cual las demandas sociales, que se transforman en políticas públicas otorgaran organicidad al aparato estatal y se materializan en instituciones, leyes, prácticas y procedimientos (Fleury, 1999). Por ello, se las puede explicar a partir del proceso que resulta de las interacciones entre los actores involucrados como también, en relación al sistema de instituciones políticas que organiza el poder de una sociedad (Repetto, 2010). En este sentido, se realza la dimensión relacional de la política social enfatizando la interacción entre diferentes actores que se enfrentan redefiniendo identidades y estrategias.

En Argentina el área de gobierno primordial para llevar a cabo la intervención en materia social (Ilari, 2006) es el Ministerio de Desarrollo Social de la Nación (MDSN). Sus políticas fomentaron una orientación territorial y principios de la economía social y solidaria con eje trabajo céntrico. Para ello, su gestión tendió a la territorialización y a la necesidad de coordinar las acciones con jurisdicciones subnacionales (Perelmiter, 2012). De este modo, generó articulación con los gobiernos municipales para que lleven a cabo implementación de programas y con Universidades Nacionales para que realicen el monitoreo.

Las orientaciones y el esquema descripto se ven reflejados en el Programa Ingreso Social con Trabajo - Argentina Trabaja (Prist - At) por la impronta territorial que configura y reconfigura el programa así como también, por su direccionalidad en términos de economía social y solidaria al formar cooperativas de trabajo. El propósito del programa era promover inclusión social a través de la conformación de cooperativas tuteladas por el Estado (Ferrari Mango, 2019b). Si bien era el MDSN quién financiaba al programa, el encargado de su ejecución a nivel territorial era el municipio. De este modo, el Prist - At desciende al territorio, a través de convenios que se firman entre el MDSN y el municipio, y se materializa a través de un instrumento: "la cooperativa".

En este marco, el objetivo general del artículo es caracterizar la dimensión relacional de la política social analizando al entramado de actores local que conforma el Prist - At y las relaciones entre ellos. El aporte del trabajo consiste en mostrar la dimensión sociopolítica en las relaciones que mantienen los actores al momento de implementarse el programa. Para ello, utilizamos una metodología cualitativa que incluyó diversas técnicas de recolección de la información como entrevistas, observación y análisis de documentos oficiales. En todas las entrevistas tuvimos el consentimiento informado aclarando el carácter anónimo, voluntario y confidencial de las mismas. El proceso de documentación, análisis e interpretación de los datos se adecuó a la intersección de proceso macro-sociales y micro-prácticas y acciones locales (Chiara y Di Virgilio, 2006; Rofman, 2010; Gonzalez Bombal, Kessler, y Svampa, 2010).

\section{Conceptualizando y reconstruyendo el entramado de actores locales}

El entramado de actores es una herramienta para reconstruir las relaciones que se forjan entre quienes participan en la ejecución de políticas y las redes de gestión que se entablan en el desarrollo de la implementación local del programa (Di Virgilio \& Galizzi, 2009). Las características del entramado varían según: número de actores, particularidades de la estructura de relaciones entre ellos, grado de institucionalización de la red, reglas de juego que regulen el comportamiento de los mismos, distribución de los recursos de poder y sus estrategias (Chiara \& Di Virgilio, 2006). Paralelamente, el entramado local da cuenta de una dimensión de carácter sociopolítico en la implementación del programa. 
Como antecedentes del análisis de actores de este programa* podemos identificar un trabajo que tiene una mirada nacional y otro que propone una caracterización a nivel local. Desde una perspectiva macro, Fernández (2012) caracterizó el entramado de actores del Prist- At a nivel nacional. El autor observó que para poner en marcha al programa hay diversos actores que intervienen en diferentes instancias del mismo: a) en la selección del distrito el MDSN; b) en la identificación de la población vulnerable el MDSN, provincial y municipal; c) en el armado de las cooperativas los entes ejecutores y son inscriptas en el registro del Instituto Nacional Asociativismo y Economía Social (INAES) y en el Registro Nacional de Efectores de Desarrollo Local y Economía Social del MDSN; d) para la aplicación de los criterios de elegibilidad interviene el MDSN, que realiza cruces con Sistema de Identificación Nacional Tributario y Social y con la administración Federal de Ingresos Públicos para verificar que se cumplan los requisitos formales de ingresos. Asimismo, el gobierno nacional firma el convenio con el Municipio. En este marco, en la implementación y supervisión del trabajo de las cooperativas participan el municipio con el INAES y Universidades nacionales designadas por el MDSN evalúan y monitorean el programa.

Asimismo, desde una visión local específicamente, en un distrito del conurbano bonaerense Fiszman (2015) recuperó la voz de diversos participantes involucrados en el programa tomando como referente empírico las reuniones de la mesa de cooperativas del Prist- At. La autora analiza las relaciones entre diversos actores tales como los referentes de las cooperativas, los funcionarios del gobierno municipal, el ente ejecutor del programa y el resto del municipio; y concluye que entre dichas relaciones circula la acumulación de poder y el crecimiento político de los referentes a partir de la implementación del programa.

Dado el alto componente territorial del programa y el recorte espacial y temporal seleccionado hemos conformado el entramado de actores del Prist- At a partir de su intervención en los Obradores del Municipio de La Matanza. Los Obradores eran inmuebles que se asemejan a galpones y están compuestos por tres espacios (Ferrari Mango, 2019a). El primer espacio es laboral en el cual se guardan los materiales de trabajo y los talleres que pueden ser de carpintería, herrería, cemento, etc. El segundo espacio es el educativo donde se dictan cursos y capacitaciones. El tercer espacio es administrativo dado que desde allí se toma la asistencia y concurrencia de los cooperativistas/ beneficiarios del programa; y también se distribuyen las tareas a realizar. En este sentido, lo Obradores eran los espacios locales de gestión desde donde se llevaba a cabo día a día la implementación del programa.

De este modo identificamos cuatro grupos de actores: funcionarios nacionales del MDSN, funcionarios del Municipio de La Matanza, técnicos de la Universidad Nacional de la Matanza (UNLaM) y beneficiarios/ cooperativistas del programa. En este sentido, desarrollaremos el marco general de cada actor que conforma al entramado. Las relaciones entre el entramado de actores se analizan considerando los siguientes ejes: las características de las relaciones entre ellos asi como también las racionalidades, los recursos de poder, los objetivos y las estrategias de cada uno. Dichas relaciones se establecen entre:

- (1) el Municipio de La Matanza con el MDSN,

- (2) el municipio de La Matanza con los cooperativistas/beneficiarios,

- (3) el Municipio de La Matanza con la UNLaM,

- (4) el MDSN con los cooperativistas/beneficiarios,

- (5) el MDSN con la UNLaM,

- (6) la UNLaM con los cooperativistas/beneficiarios.

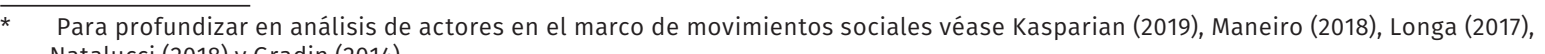
Natalucci (2018) y Gradin (2014). 


\section{Caracterización del entramado de actores}

\subsection{Gobierno Nacional: Ministerio de Desarrollo Social de la Nación}

En 1994 durante la presidencia de Carlos Menem, se creó la Secretaría de Desarrollo Social. La misma se transformó de Secretaría a Ministerio de Desarrollo Social y Medio Ambiente (Paura, V Zibecchi, 2014). Su objetivo era asistir al Presidente de la Nación y al Jefe de Gabinete de Ministros en la promoción y asistencia social.

Desde el año 2003 hasta el año 2015, el MDSN trazó dentro de los ejes de la política social fortalecer la territorialidad de la misma canalizando la participación de los actores locales y fomentando la economía social con una visión trabajo céntrica. En este sentido, la gestión planteó la necesidad de un ministerio que trabaje directamente en el territorio saliendo de los escritorios (Perelmiter, 2012) a favor de una política que genere inclusión y participación (De Piero, 2015).

En este sentido, las estrategias impulsadas por el MDSN tuvieron principios de la economía social y solidaria y una visión trabajo céntrica. También, se ha revitalizado el cooperativismo de trabajo a través de la recuperación de empresas por parte de sus trabajadores o bien a través de políticas públicas que conforman cooperativas de trabajo bajo la tutela del Estado (Rebón \& Kasparian, 2015). En lo que refiere a la gestión del ministerio, caracterizada por una fuerte tendencia a la territorialización se observó el despliegue de agencias del ministerio, que establecieran materialmente su presencia en todo el territorio nacional y la necesidad de coordinar las acciones con las jurisdicciones subnacionales (Perelmiter, 2012: Binazzi, 2019). En el territorio se entrecruzan las construcciones políticas, los destinatarios de sus políticas llevan a cabo su vida cotidiana actuando política y/o socialmente, y los sentidos de pertenencia ya no ancladas en lo nacional. En este caso, la presencia física en el territorio del MDSN toma corporalidad intermitentemente a través de los agentes territoriales que ocasionalmente visitan los Obradores. Ellos, con un previo acuerdo con el funcionario técnico municipal, concuerdan un recorrido para mostrarles los resultados del trabajo que vienen desarrollando.

\subsection{Gobierno Municipal: Municipio de La Matanza}

Los municipios, como nivel jurisdiccional dentro del sistema federal argentino, son el nivel de gobierno más próximo a la sociedad y al ser la cara más directa e inmediata de lo público, de lo estatal, es receptor y canalizador de las nuevas demandas sociales y responde a las mismas con mayor flexibilidad y comprensión, convirtiéndose en articulador y coordinador de redes de gestión tanto públicas como privadas (Herzer y Piréz, 1990 y Bottini, 2009).

La reforma del Estado que atravesó a los países latinoamericanos, y particularmente a Argentina en la década del noventa, estuvo inspirada en un proceso de descentralización que reconfiguró las competencias asignadas al Estado, sociedad y mercado produciendo una nueva división del trabajo (Oszlak, 1997). Se produce una suerte de revitalización de la esfera local y el municipio aparece como punto de condensación de la fragmentación social, de la protesta, de la crisis de mediaciones y de la falta de recursos (García Delgado, 1996; Arroyo, 1997; Iturburu, 2000; Cravacuore, 2007; Chiara y Di Virgilio, 2006).

En efecto, se produjo la jerarquización del espacio local provocado por el reordenamiento de competencias, atribuciones y responsabilidades estatales constituyéndose como ámbito de desarrollo e instancia privilegiada para implementar políticas sociales (García Delgado, 1996; Arroyo, 1997). Si bien 
el Prist-At es un programa nacional, no se implementó en la totalidad del país, sino que se priorizan provincias en función de dos cuestiones: los requerimientos del Programa (población objetivo/criterios de elegibilidad) y las condiciones institucionales, las capacidades y posibilidades de gestión de los entes ejecutores. Nuestro recorte espacial fue el Municipio de La Matanza, que es uno de los 134 municipios la Provincia de Buenos Aires (la provincia tiene una población 15.594.428), el más extenso del conurbano con una superficie total de 325,71 kilómetros cuadrados, limita al noroeste con la Ciudad Autónoma de Buenos Aires y según el Censo 2010 realizado por el INDEC, posee una población que asciende a 1.775.816 habitantes de los cuales 866.690 son varones y 909.126 son mujeres.

El mismo concentra el 12\% de los electores de la provincia, tornándose su control absolutamente clave para la dinámica política subnacional. De este modo, se comprende y ejemplifica una de las funciones de la política social, considerada como legitimadora del partido gobernante y del sistema en su conjunto, al conseguir votos, mantener la paz y la armonía social (Ilari, 2006). Dada la importancia, que se le asigna a este elemento, es otro de los motivos por los nos centramos como referente empírico al Municipio de La Matanza.

Para llevar a cabo la implementación del Prist - At en el distrito de la Matanza, el municipio tuvo que reorganizar su organigrama. De este modo, la cadena de mando quedo conformada por los encargados de los Obradores, seguido de la secretaría de unidad ejecutora del programa que depende directamente de la intendencia municipal.

\subsection{Universidad Pública: equipo técnico de la Universidad Nacional de La Matanza}

La naturaleza y misión de las universidades se ha puesto en debate desde los primeros años del siglo XXI en consonancia con la problematización más amplia y abarcativa acerca del rol del Estado (Castronovo \& Lischetti, 2013). Desde el año 2004 el Estado Nacional les demanda a las Universidades Públicas más asistencia técnica, investigaciones conjuntas o direccionadas para dar respuesta a interrogantes que la propia gestión pública necesita responder (Castronovo \& Lischetti, 2013), la cooperación y la transferencia, como una forma de contribuir a los procesos de desarrollo local y regional, en temas productivos, ambientales, tecnológicos, salud, empleo, economía social, gestión y servicios públicos (García Delgado \& Casalis, 2013).

En este maro, las Universidades Nacionales han firmados convenios más asiduamente con el Estado en diferentes materias destacando el carácter de consultoras preferenciales que otorga el Estado a las Universidades Nacionales. Dichos convenios las hace partícipe en alguna de las etapas del ciclo de las políticas públicas sea la formulación, implementación o bien una instancia de evaluación. En lo que refiere al Prist-At, las Universidades públicas han tenido dos roles: capacitación de titulares del programa y monitoreo del mismo. Las capacitaciones se han desarrollado con un alto contenido en los ejes que centro la gestión del MDSN: economía social y solidaria y territorialidad. En este trabajo, nos interesa particularmente el rol de la UNLaM en el monitoreo del programa.

La Universidad Nacional de La Matanza se creó en el año 1989 y está ubicada en la localidad de San Justo del Municipio de La Matanza en la Provincia de Buenos Aires en una de las zonas con mayor densidad poblacional del país. Se caracteriza por ser una de las universidades que se creó en el conurbano bonaerense en las últimas décadas, específicamente en el distrito de La Matanza. La UNLaM ha celebrado convenios para brindar capacitaciones a los titulares del programa pero en este trabajo nos interesa su rol para monitorear el Prist-At en calidad de técnico y asesor en la cotidianeidad de la implementación del programa. 
Para llevar a cabo la tarea de monitoreo, la UNLaM contaba con un equipo de gabinete que realiza funciones técnicas y administrativas y un equipo territorial que jerarquiza los puestos en coordinador, supervisor y auditor. En lo que refiere al equipo técnico territorial de la Universidad, en los Obradores se encontraban los auditores cuya tarea era registrar la asistencia. Paralelamente, la supervisora recorría los diversos Obradores para corroborar la asistencia de los auditores y observar que estén trabajando sin ningún inconveniente así como también elaboraban un informe semanal que remite a su coordinadora. Por último, los coordinadores asistían a los supervisores y tienen contacto directo con la unidad ejecutora de las cooperativas a cargo del municipio, para rendir cuentas de la parte económica de la "cooperativa". A su vez, recolectan los informes de los supervisores y luego elaboran uno final y lo envía a ministerio.

\subsection{Titulares del programa: Cooperativistas / Beneficiarios}

El programa perseguía formalmente que el cooperativista/beneficiario se dignifique a través del trabajo. Para ello, se conformaron cooperativas de trabajo bajo la tutela del Estado que les permitieran realizar una contraprestación laboral e incluso formación integral y capacitaciones. De este modo, los cooperativistas/ beneficiarios están subjetivados por la condicionalidad que interpone el Prist -At para ingresar al mismo así como también para poder permanecer debían realizar una contraprestación. La misma consistía en obras de mediana y baja complejidad, con impacto directo en los barrios postergados, tales como tareas involucran saneamiento, infraestructura urbana, mejoramiento de espacios verdes, infraestructura comunitaria y viviendas.

El perfil sociodemográfico de los beneficiarios se caracteriza por ser en mayor medida mujeres, el componente etario mayores de 18 a 24 años (30\%) pero el 42\% tiene más de 35 años, la mayoría curso el secundario y más de la mitad lo ha finalizado. En lo que refiere al ámbito laboral más del $70 \%$ no ha tenido oficio ni profesión previa al ingreso al programa y el 53\% de los desempleados al momento de su ingreso al programa llevaba más de 6 meses sin trabajar. A agosto de 2015 el Prist - At cuenta con un total de 218.870 titulares a nivel nacional (Nación, 2015). Si bien no hay datos concretos de la cantidad de titulares del programa en el Municipio de La Matanza Nahuel, funcionario municipal nos cuenta que existen alrededor de 10.000 cooperativistas/ beneficiarios en el distrito.

A nivel territorial, dentro del grupo de los cooperativistas encontramos varias clasificaciones: cooperativista, cooperativista-capataz, cooperativita-encargado de pañol, cooperativista-encuestador y cooperativista-orientador. Los cooperativistas son aquellos que hacen la labor de servicio o de producción que le es asignada por el funcionario municipal, es decir, son aquellos cooperativistas que realizan las actividades laborales que demandan el programa (Fouskas, Gikopoulou, Ioannidi y Koulierakis, 2019). El cooperativista-capataz es el encargado de un determinado taller y por lo cual en el mismo capacita a los cooperativistas que tiene a su cargo en la actividad productiva de ese taller sea herrería, carpintería u hormigón. Si bien es cooperativista se lo conoce como la figura del capataz. Él es quién capacita y supervisa la labor del resto de los cooperativistas. En lo que refiere al cooperativista-orientador, es aquél que se encarga de tomar la asistencia de sus compañeros, recibir certificados por ausencias y luego lleva toda la documentación al ente ejecutor del municipio. Entre sus compañeros lo llaman planillero. Esa planilla es una hoja que tiene las siguientes columnas: nombre, apellido, DNI y firma del cooperativista. Los cooperativistas - encargados de pañol son los que se encargan de verificar y entregar los materiales y herramientas. Los cooperativistas- encuestadores, aplican diversos cuestionarios a la comunidad local sobre algún tema específico. 
De este modo, hay una primera subjetivación del programa hacia los beneficiarios en tanto las condicionalidades que se establecen para ingresar al mismo. Luego, hay una segunda subjetivación que responde a la permanencia del cooperativista/ beneficiario en el programa en tanto sujeto apacible del programa que pretende dignificarlo a través del trabajo. Finalmente, existe una tercera subjetivación del cooperativista/beneficiario que está vinculada a su rol en el programa y se va transformando y consolidando en función de la tarea que le es asignada.

\section{Análisis del entramado de actores}

\subsection{Racionalidades y características de las relaciones entre los actores}

En el proceso de implementación de políticas públicas se desarrollan sucesivas tomas de posición del Estado y de actores sociales que reproducen concepciones y modelos sobre formulación e implementación generando contradicciones al momento de materializarlas. En este marco, se establece una relación entre la política y la administración (Wilson, 1980; Gonzalez Mercado, 2005 y Thwaites Rey, 2001). A la primera, se la entiende como la actividad que permite formular y planificar las políticas públicas con una "bondad intrínseca", mientras que la segunda, adquiere una "forma oblicua" tras su implementación a través del aparato burocrático (Oszlak, 1997). La explicación del autor refleja la relación entre el Municipio de La Matanza y el MDSN. El municipio, en calidad de ente ejecutor, "renuncia" a implementar diligentemente objetivos y programas formulados por legisladores o técnicos que manejan un conocimiento adecuado, pero carecen de poder suficiente para imponer sus propuestas. El MDSN, al descentralizar la implementación preserva la legitimidad de la institución auspiciante.

En este sentido, los dos modelos que presuponen formas muy diferentes de hacer política, es decir, formular e implementar políticas públicas: el "incrementalista" (Lindblom, 1959) y el "optimalista" (Dror, 1964). En el primero la razón subordina a la acción que pretende lograr determinados fines a través de los medios más racionales. Su forma extrema, está representada por la planificación que orienta la acción social hacia la satisfacción de determinados objetivos mediante la aplicación de criterios de racionalidad técnicos. Este modelo se asemeja más a la orientación del MDSN al formular el Prist -At. El segundo denominado interacción, explica que la acción es producto de transacciones entre partes donde el interés individual de cada uno realiza el fin colectivo que las vincula. Para este modelo, el intercambio y la negociación son los elementos característicos de la acción política. Dicho modelo, se asemeja más a la modalidad de implementación que lleva a cabo el municipio.

A modo de ejemplificación, Lucas en calidad de coordinador del equipo técnico de la UNLaM, señala cómo se produce un cambio entre la formulación e implementación del programa.

\footnotetext{
Hay una hay una normativa, tiene una naturaleza, pero en el momento de su implementación va mutando es esa idea, o esa política pública. Y porque tiene que ver con los actores locales. En principio las necesidades del Municipio. No siempre el creador o el pensador de la política pública tienen un conocimiento puro del territorio. Hay una idealización. Si uno presta atención a la normativa madre de la política pública y ve los escritos del Ministerio, hay una cuestión medio utópica. (Lucas, técnico UNLaM).
}

De este modo, la verbalización de Lucas recupera como el Prist - At si bien tiene una normativa, se va transformando y reconfigurando en la implementación del programa a partir de un entramado de actores. En los Obradores, se visualiza la idea de darle al programa una perspectiva más "productiva" pero en la práctica el propósito requiere tiempo y a su vez, voluntad del encargado del Obrador como así también de los cooperativitas que son quienes llevarían a cabo tareas con perspectiva productiva. 
En efecto, Lucas hace hincapié en la mutación del programa fundamentalmente por la intervención de los actores. A partir de aquí, nos interesa vislumbrar que características adquieren el entramado de actores del Prist -At en el distrito de La Matanza, específicamente las particularidades de la estructura de relaciones entre los actores que lo componen. Las relaciones se establecen entre: (a) municipio de La Matanza con MDSN, (b) municipio de La Matanza con los beneficiarios/cooperativistas, (c) municipio de La Matanza con la UNLaM, (d) MDSN con los cooperativistas/beneficiarios, (e) MDSN con la UNLaM y (f) cooperativistas/beneficiarios con la UNLaM.

En lo que refiere a la relación entre el MDSN y el municipio, Lucas señala:

\begin{abstract}
Es tensa. El que, el Ministerio nunca confía plenamente en el Municipio. Aunque sea compañero, aunque sean del mismo palo, no confía. ¿Por qué? Porque son dos tipos distintos de hacer política. El municipal tiene que ver con una tradición... diga, digamos, de baja profesionalización, de baja tecnificación, y de poca intervención estatal, y de una intervención en territorio que tiene que ver más que nada con la política partidaria. De, una lectura de los movimientos sociales, de los sectores vulnerables muy fuerte. (Lucas, técnico UNLaM)
\end{abstract}

De este modo, la primera descripción de la relación entre MDSN y Municipio es descripta por Lucas como "tensa". Dicha tensión, se observa en conversaciones con diferentes funcionarios municipales. Ellos identifican a los técnicos de la UNLAM como "aquellos que vienen a controlarlos" por ordenamiento y desconfianza de los agentes del MDSN. Asimismo, Lucas agrega que:

\footnotetext{
No siempre es buena porque son dos lógicas distintas de política. Son dos lógicas completamente de política. En términos operativos, hay una cuestión programática y una cuestión ideológica. La política del Conurbano es una política conservadora. La política del Ministerio, si vos lees los programas es una política mucho más progresista. (Lucas, técnico UNLaM)
}

De este modo, Lucas menciona dos lógicas diferentes de hacer política que permiten vislumbrar las racionalidades que priman en cada uno de estos actores. Dichas lógicas, están guiadas por la racionalidad técnica y la racionalidad política. La primera, refiere a los fines-medios de la política pública plasmada a través de una racionalidad técnica en la formulación del programa. Específicamente, en la normativa que le dio origen y en los convenios firmados entre el intendente del Municipio y el representante del MDNS. La segunda, se alimenta del interés por obtener acumulación política y legitimidad.

Asimismo, al interior del nivel municipal también está presente la tensión y retroalimentación entre la racionalidad política y técnica. Nahuel, ejemplifica las pretensiones de la intendenta y cómo logra articular el escenario deseado con la realidad de las capacidades de los cooperativistas:

\footnotetext{
Se hicieron reuniones. Vamos y venimos, conversamos, nos peleamos, volvemos para acá, [me dicen] "no, esto no se puede hacer". Viene el secretario de la intendenta y me dice que quiere que construyamos un puente para ir a la Luna", yo le digo que no se puede y me dicen "bueno, pero lo tenés que hacer porque lo pidieron", etc. Claro, es así. Entonces, este es el ida y vuelta. (Nahuel, funcionario técnico municipal)
}

Recuperando la narrativa de Nahuel, se observa su intención de realizar una planificación en torno a los objetivos que puede lograr a partir de los recursos con los que cuenta. En este marco, existe una negociación entre los agentes del MDSN con el municipio de la Matanza para establecer las bases de las actividades que deben llevar a cabo los cooperativistas. Asimismo, la UNLaM funciona a modo de nexo entre ambos al realizar el monitoreo del Prist-At.

En lo que refiere a la relación de la UNLaM con el municipio, en un comienzo era conflictiva dado que los funcionarios del municipio percibían control y vigilancia por los técnicos de la universidad. A su vez, también se entremezclaban dos tipos de racionalidades. El primero, fomentaba una racionalidad técnica mientras que el segundo una racionalidad política. 
En la implementación del programa, día a día, auditores y supervisores representaban a la UNLaM y se vinculaban con los funcionarios del municipio o delegados. La racionalidad técnica de la UNLaM utilizaba mecanismos de negociación en el territorio. Dicha negociación, entre los funcionarios del municipio y el equipo técnico de la UNLaM, se ve reflejada en la narrativa de Lucas:

\begin{abstract}
Yo tenía que velar por que todos los días se pudiesen tomar asistencia. Para eso me quedaba hablando con los delegados, hablando con el referente político, alguien de la cooperativa. Yo me llevaba muy bien con ellos porque negociaba de antemano cuál era el límite entre mates, risas, hablando de política. Vos vas viendo que al tipo se le va transformando la cara, listo: mañana intentaré llegar un poquito más. “Che, y por qué, escúchame: ¿cuántas personas son?" "Cien" “¡Y por qué están ahí noventa y ocho? ¿Dónde están las otras dos?”. O sea, todos los días vas tocando el terreno. No tenía problemas. Era un pacto. (Lucas, técnico UNLaM)
\end{abstract}

De este modo, en la narrativa de Lucas toma presencia la dinámica política en el territorio tras establecer desde el inicio los límites y generar un acuerdo. No obstante, dichas negociaciones eran solamente con el fin último de poder realizar su rol técnico en el programa. En este sentido, se realza el rol técnico de la Universidad. En lo que refiere a la relación entre la UNLaM y el MDSN, está determinada por un convenio de asistencia técnica. Lucas detalla cómo es el vínculo entre la universidad y el ministerio de la siguiente manera:

Las reuniones con gente del Ministerio son sobre agenda, de trabajo. La universidad tiene un convenio con una cláusula de confidencialidad. Toda la información que genera la universidad respecto al Programa Argentina Trabaja es netamente confidencial. Dice a ver: cuáles son las tareas y cuáles son las prerrogativas. Es un contrato. (Lucas, técnico UNLaM)

En este marco, una de las características que adquiere el vínculo entre la UNLaM y el MDSN a través de la formalidad del convenio es la de confidencialidad. Como consecuencia del mismo, la relación entre el MDSN y el cooperativista/beneficiario del programa se encuentra mediatizada por el funcionario municipal y el equipo técnico de la UNLaM.

En algunos casos, el cooperativista reconocía que el programa estaba financiado desde Nación, pero existía una confusión al ser el municipio la cara visible en la implementación. De este modo, el nexo producto de la modalidad del convenio entre el MDSN y el cooperativista no era frecuente. Excepcionalmente, agentes territoriales visitaban los obradores o los puntos de citación para verificar que el trabajo se estaba realizando.

En este sentido, la relación entre el cooperativista y los funcionarios municipales era frecuente. El vínculo se caracterizaba por ser diario y los funcionarios municipales eran los que estaban al tanto de sus necesidades, capacidades y preferencias dado que ellos dividían las tareas, tal como lo expliqué en el capítulo anterior. Lo mismo sucedía en la relación entre el cooperativista y el equipo técnico de la UNLaM porque los auditores les tomaban la concurrencia todos los días. Lucas refiere a dicho vínculo:

\footnotetext{
Una forma de vinculación distinta con el asociado. Una confianza. En el Conurbano lo que nos pasaba es que bajaba gente del Ministerio y si nosotros estábamos presentes, los cooperativistas miraban a la gente del Ministerio y después nos miraban a nosotros y nosotros les decíamos que sí. Nosotros éramos como el factor legitimador. Se genera una legitimidad...se genera legitimidad. La universidad construyó legitimidad. Porque siempre lo que dijo la universidad. Netamente programático y operativo. (Lucas, técnico UNLaM)
}

De este modo, se observa que los lazos entre los cooperativistas y el equipo técnico de la UNLaM se caracterizan por un vínculo de confianza. Dicha confianza, está inspirada en el respeto que deviene de la UNLaM en tanto institución formativa y principalmente por esa cotidianeidad que construía el técnico de la UNLaM en la dinámica territorial de la implementación de programa. En definitiva, el técnico realizaba su trabajo al igual que el cooperativista y este último lo veía como un referente frente a alguna cuestión problemática que atravesara. 
Asimismo, otro de los aspectos que permite caracterizar el entramado es la estabilidad entre las relaciones de los actores y los grados de institucionalización de la red que entablan. En términos formales, la estabilidad está dada por el cumplimiento de convenio. El MDSN celebró los convenios con el Municipio para que llevara a cabo la implementación y en el mismo se establecían la cantidad de cooperativistas/ cooperativas que podían formar parte del programa. Paralelamente, firmó convenio con la UNLaM para que realicé el monitoreo del programa. No obstante, tras el cambio de gobierno el MDSN no renovó el convenio con la UNLaM y antes de volver a celebrar el acuerdo con el municipio se aseguró de recibir informes que presenten los resultados obtenidos. Por lo tanto, el grado de estabilidad e institucionalización de la red está supeditado a la renovación de los convenios.

En la cotidianeidad del programa, los funcionarios municipales, especialmente los encargados de los Obradores, gozan de un contacto directo con los técnicos de la UNLaM y con los cooperativistas/beneficiario. Los funcionarios municipales les asignan las tareas a los cooperativistas y le controlan el presentismo, como así también identifican problemas personales que poseen, generando una familiaridad. Dicha familiaridad, también está presente en la relación ente los técnicos de la UNLaM y el cooperativista/ beneficiario. De forma contraria y términos generales, se puede indicar que los agentes del MDSN no cuentan con contacto directo con los cooperativistas/beneficiarios sino que la vinculación está mediatizada por la UNLaM o bien por los funcionarios en calidad de representantes locales. Tanto el equipo técnico de la UNLaM como los funcionarios municipales poseen vinculación a través de reuniones, capacitaciones, pedido de informes y listados de concurrencia de los cooperativistas.

4.2 Lógicas de acción, recursos de poder, objetivos y estrategias de los actores

Para continuar analizando el entramado de actores nos concentramos en las reglas de juego que regulan su comportamiento, la distribución de los recursos de poder, objetivos y estrategias que llevan a cabo. Los diferentes tipos de recursos que otorgan relativas dosis de poder a individuos o colectivos pueden ser: de poder político y social, de poder económico y de poder técnico-administrativo o bien cognitivo y simbólico (Di Virgilio \& Galizzi, 2009). Según la dotación con que cuentan los diferentes tipos, podrán negociar o ejercer acciones de veto en los ámbitos de interacción (Repetto, 2010).

Si bien los actores poseen, construyen y adquieren diferentes tipos de recursos de poder algunos predominan sobre otros. En el caso del municipio de La Matanza, los que predominan son sociales y políticos. A este recurso de poder social, lo entendemos como aquel que contribuye a entablar redes con la comunidad local en función de dinámicas territoriales caracterizadas por vínculos de proximidad y cotidianeidad. El recurso de poder político está presente en el MDSN dada su legitimidad de origen y trayectoria en fomentar programas sociales. El otro recurso de poder que predomina en este actor es el económico, dado que el programa se financia en gran medida con fondos provenientes del mismo.

En lo que respecta a los recursos de poder de la UNLaM, éstos se encuentran asociados al conocimiento técnico-administrativo, cognitivo y simbólico. Los mismos, se fundamentan en su trayectoria en materia de formación de recursos humanos y fomento a la investigación. Asimismo, en los últimos años adquirió un mayor protagonismo tras promover estrategias para vincularse con las problemáticas del territorio circundante. Dicho recurso, se visualiza en el respeto y confianza que le tienen los cooperativistas/ beneficiarios al equipo técnico de la UNLaM. 
Finalmente, los cooperativistas tienen recursos de poder social y político. El primero, se fundamenta en su conocimiento sobre la comunidad local, es decir, vecinos, instituciones u organizaciones sociales. Dicho conocimiento, le permite enmarcarse en una trama local caracterizada por redes y dinámicas territoriales. A modo de ejemplo, las personas que se inscribieron en el programa se enteraron a través de vecinos o en alguna institución del barrio. En lo que respecta al recurso de poder político, son los representados que tras su voto legitiman a los representantes. A su vez, son fundamentales en las movilizaciones que buscan garantizar apoyos o demostrar su disconformidad con determinada política pública o situación socioeconómica. De este modo, este tipo de recurso de poder político se visualiza en los operativos territoriales y en las movilizaciones que explique anteriormente.

Para la implementación del programa, el MDSN tuvo como estrategia establecer al municipio como ente ejecutor por su proximidad territorial para acceder rápidamente a los posibles beneficiarios. A su vez, complementó esta decisión adjudicándole a la UNLaM la función de monitorear el programa con el fin de verificar que el municipio no haga un "mal uso" del mismo y para otorgarle mayor transparencia y legitimidad dado que la universidad es una institución respetada por el conjunto de la sociedad.

Ambas estrategias, se argumentan a partir del recurso que posee cada uno de estos actores. Los funcionarios del Municipio de la Matanza cuentan con recursos políticos y sociales mientras que la UNLaM tiene con recursos simbólicos, cognitivos y técnico-administrativo. La estrategia del MDSN, generó en un principio una relación hostil entre los funcionarios del municipio y los técnicos de la UNLaM porque los primeros percibían que los segundos tenían la función de controlarlos.

El Municipio de la Matanza persigue como objetivo incrementar sus capacidades técnico-administrativas, mejorar la infraestructura del municipio, mejorar la calidad de vida de la comunidad, obtener recursos por parte de MDSN para aplicar en su territorio y capitalizar a los beneficiarios a modo de acumulación política. Los funcionarios municipales emplean el Prist-At como estrategia para aumentar la acumulación política.

Es decir, si bien en varias entrevistas los funcionarios municipales reconocen que el programa es nacional e incluso mencionan al MDSN como "la madre del programa", logran capitalizar el programa dándole un tinte propio territorial que denota un compromiso entre el funcionario político municipal con el cooperativista. Dicha estrategia de acumulación política también es propia del MDSN, quien sanciona la resolución del Prist -At en un contexto sociopolítico adverso.

En el caso de la UNLaM, capitaliza su rol de técnica en el monitoreo del programa permitiendo brindar trabajo a estudiantes próximos a recibirse o bien jóvenes graduados. A su vez, les permite contribuir con su compromiso social vinculándose con el territorio y fortalecer su legitimidad. Para poder realizar su trabajo en el territorio, tuvo que aprehender dinámicas territoriales y generar un vínculo tanto con funcionarios municipales como con cooperativistas.

Por último, los cooperativistas tenían como objetivo bregar por sus necesidades socioeconómicas recibiendo un ingreso económico y enfatizando su inclusión a través del mecanismo de trabajo cooperativo. De este modo, se vislumbra que los cooperativistas se benefician de las posibilidades de obtener capacitaciones, aprender oficios o bien cursar el secundario. Asimismo, interactúan con auditores de UNLaM para resolver problemáticas sociales. Paralelamente, participan de movilizaciones propuestas por MDSN y Municipio. 


\section{Conclusiones}

En este artículo se analiza la política social desde una mirada relacional tomando como eje el entramado de actores de un programa que adquirió relevancia en la Argentina. Al centrarnos en el entramado de actores, se puede identificar la modalidad que adquiere el programa a medida que se implementa influenciado por sujetos colectivos sociales y estatales con intereses, demandas y estrategias.

El entramado de actores nos permitió observar principalmente dos cuestiones. Por un lado, que el conjunto de actores dentro de cada grupo no es homogéneo, sino que en virtud de sus funciones se los subjetiviza de modo diferente. Por otro lado, se atribuye un rol a cada actor en la formulación del programa desde el MDSN. Dicha atribución, nos llevó a preguntarnos por qué el municipio era el ente ejecutor en el territorio, por qué la universidad era la que monitoreaba el programa y por qué los beneficiarios del programa se los consideraban cooperativistas. Las respuestas a estas preguntas fueron halladas a través de las dimensiones tales como tipo de recurso de poder que prevalece en cada actor y el tipo de racionalidad predominante que guía su acción, objetivos e intereses.

Los agentes del MDSN, al momento de formular el programa consideraron su limitación espacial, es decir, su escasa capacidad de despliegue en el distrito para acceder a los sujetos. Por ello, le asignaron al municipio la implementación dado que contaba con conocimiento del territorio, la relación de proximidad con los posibles participantes y el aparato estatal con capacidad de desplegarlo en el territorio local. Paralelamente, el MDSN firmó convenios con la UNLaM para que lleve a cabo el monitoreo del programa. Por lo cual, tanto funcionarios municipales como técnicos de la UNLaM reconocían que la universidad cumplía un rol de control para con los municipios.

En el entramado de actores locales, al momento de la implementación del programa, se entremezclan tipos de racionalidades técnicas y políticas así como también recursos, estrategias y objetivos. En el caso de los técnicos de la UNLaM prima la racionalidad técnica, en lo funcionarios municipales la política, mientras que los funcionarios del MDSN la técnica, aunque subyace la racionalidad política. En lo que refiere a los recursos, en los funcionarios del municipio prima poder social y político, en agentes gubernamentales del MDSN se resalta poder político y económico, en funcionarios técnicos de la UNLaM se destaca el simbólico, cognitivo y técnico-administrativo y finalmente, en los cooperativistas predominan el poder social y político. En definitiva, los recursos están relacionados al rol que cumple cada actor en el programa, a las características que adquieren las relaciones entre ellos, sus objetivos y estrategias.

En definitiva, las interrelaciones entre actores en la implementación materializan y reconfiguran al programa en el territorio. Paralelamente, contribuyen a incrementar su acumulación de poder y capacidad de agencia.

\section{Referencias bibliográficas}

Arroyo, D. (1997). Estilos de gestión y políticas sociales municipales en Argentina. En D. García Delgado (ed.), Hacia un nuevo modelo de gestión local, Municipio y Sociedad Civil en Argentina. pp. 315-328. Buenos Aires: Universidad de Buenos Aires

Bottini, A. (2009). Ejercicio pleno de la Autonomía Municipal en la Provincia de Buenos Aires ¿Se puede propender a la descentralización Político-Institucional de las Municipalidades Bonaerenses por medio de Actos Legislativos Emanados de los propios Municipios? Ponencia presentada en el X seminario de la Red Muni, Buenos Aires. 
Binazzi, A. (2019). Género y migraciones forzosas en Centroamérica y el Caribe. Una mirada antropológica para los derechos humanos de las niñas, adolescentes y de las mujeres. Collectivus, Revista de Ciencias Sociales, 6(1), 155-176. DOI: http://dx.doi.org/10.15648/Coll.1.2019.9

Castronovo, R., \& Lischetti, M. (2013). Universidades latinoamericanas: Compromiso, praxis e innovación. Buenos Aires: Editorial de la Facultad de Filosofía y Letras Universidad de Buenos Aires.

Chiara, M., \& Di Virgilio, M. (2006). La política social en la crisis de la convertibilidad (1997 -2001): mirando la gestión desde las coordenadas municipales en el Gran Buenos Aires. In L. Andrenacci (Ed.), Problemas de la política social en la Argentina contemporánea. (pp. 125-156). Buenos Aires: Prometeo.

Cravacuore, D. (2007). Los municipios argentinos (1990-2005)". En Cravacuore, D. e Israel R. (Comp.). Procesos políticos municipales comparados en Argentina y Chile (1990-2005). Buenos Aires, Argentina: Editorial de la Universidad Nacional de Quilmes-Universidad Autónoma de Chile.

De Piero, S. (2015). Una propuesta de indicadores de representación social en políticas sociales en territorio. Congreso SAPP, Mendoza.

Di Virgilio, M., \& Galizzi, D. (2009). Los actores en el entramado de la gestión de la política social: una aproximación conceptual y elementos para el análisis. In M. Chiara \& M. M. Di Virgilio (Eds.), Gestión de la política social. Conceptos y herramientas (pp. 319-345). Buenos Aires: Prometeo Libros-Universidad Nacional de General Sarmiento.

Dror, Y. (1964). Muddling Through-"Sience" or Inertia. Public Administration Review, 24(3), 153-157.

Fernández, J. (2012). La implementación de programa Ingreso Social con Trabajo "Argentina Trabaja" en ámbitos subnacionales. In Documento de Trabajo no 96. Buenos Aires: CIPPEC.

Ferrari Mango, C. (2019b). Política Social desde Cooperativas Reguladas: Argentina Trabaja-Programa Ingreso Social Con Trabajo. Revista de ciencias sociales de la Universidad de Costa Rica. 165 (III-2019).

Ferrari Mango, C. (2019a). Territorio, política social e implementación. Una mirada desde los espacios locales de gestión del Argentina Trabaja - Programa Ingreso Social con Trabajo en el distrito de La Matanza. Ciudadanías. Revista de Políticas Sociales Urbana No 4 primer semestre de 2019.

Fiszman, L. (2015). Disputas en torno al trabajo político: cooperativas, militancia y reconocimiento en el Programa Ingreso Social con Trabajo en un municipio bonaerense. In P. Formi \& L. Castronuovo (Eds.), Ni punteros ni piqueteros. Organizaciones populares durante el kirchnerismo (pp. 69-98). Retrieved from https://www.flacso.org.ar/publicaciones/ni-punteros-ni-piqueteros/

Fouskas, T., Gikopoulou, P., Ioannidi, E., y Koulierakis, G. (2019). Gender, transnational female migration and domestic work in greece: an intersectional review of research on female migrants' access to labour, healthcare and community associations. Collectivus, Revista de Ciencias Sociales, 6(1), 99-134. http://dx.doi. org/10.15648/Coll.1.2019.7

Fleury, S. (1999). Políticas sociales y ciudadanía. Banco Interamericano de Desarrollo, Instituto Interamericano Para El Desarrollo Social (INDES), 1-28. Retrieved from https://consejopsuntref.files.wordpress.com/2017/08/ fleury.pdf

García Delgado, D. (1996). Nuevos escenarios locales. El cambio del modelo de gestión. In Daniel García Delgado (Ed.), Hacia un nuevo modelo de gestión local. Municipios y sociedad civil en Argentina (p. 13). 
Retrieved from https://biblio.flacsoandes.edu.ec/catalog/resGet.php?resld=43200

García Delgado, D, \& Casalis, A. (2013). Modelo de desarrollo y universidad en Argentina. Análisis crítico y contribución de la extensión universitaria al desarrollo local y regional. Revista de Extensión Universitaria de La UNL, (3), 24-31.

Gonzalez Bombal, I., Kessler, G. y Svampa, M. (2010). Introducción. Las reconfiguraciones del mundo popular. En Kessler, G.; Svampa, M. y Gonzalez Bombal, I. (comp) (2010). Reconfiguraciones del Mundo popular: El Conurbano Bonaerense en la posconvertibilidad. Buenos Aires: editorial Prometeo - Universidad Nacional de General Sarmiento.

González Mercado, J. (2005). La Administración Pública como Ciencia: una aproximación a su objeto de estudio. Espacios Públicos, 8 (15), 162-175.

Gradin, A. (2014). Estado y mediaciones sociales: El estilo de gestión del Movimiento de Desocupados Barrios de Pie en el territorio (Argentina 2002-2011). Estudio digital, (32), 49-78.

Kasparian, D. (2019). Promoción estatal del cooperativismo de trabajo y formas socioproductivas emergentes. Contribuciones en clave emancipatoria a partir de un estudio de caso del Programa Argentina Trabaja. RevIISE | Vol. 13, Año 13 | abril 2019 - septiembre 2019 pp. 211-225.

Herzer, H., \& Piréz, P. (1990). Gobierno Local. In N. Clichesvsky (Ed.), Construcción y administración de la ciudad latinoamericana (pp. 175-191). Buenos Aires: Grupo Editor Latinoamericano.

Ilari, S. (2006). Entre el género y la especie. Reflexionando sobre la naturaleza de la política y la Gestión social. Revista Circunstancia, Instituto Universitario de Investigación Ortega y Gasset, Año IV, (11).

Isuani, E. (2008). La política social argentina en perspectiva. En G. Cruces (Ed.). Los programas sociales en Argentina hacia el Bicentenario. Buenos Aires: Banco Mundial.

Iturburu, M. (2000). Municipios Argentinos. Fortalezas y debilidades de su diseño institucional. Buenos Aires: Documento de trabajo, INAP.

Lindblom, C. (1959). La ciencia de abrirse paso. In Clásicos de la Administración Pública. Mexico: Edición FCE.

Lischetti, M. (2013). Introducción. In M. Lischetti (Ed.), Universidades latinoamericanas: compromiso, praxis e innovación (pp. 13-22). Buenos Aires: Editorial de la Facultad de Filosofía y Letras Universidad de Buenos Aires.

Nación, M. de D. S. de la. (2015). Situación actualizada de las cooperativas mixtas perfil de los titulares y aspectos evaluativos al primer semestre 2015.

Natalucci, A. (2018). El neoliberalismo en acto: políticas sociales y experiencias organizativas en Argentina (2009-2016). Polis (Santiago), 17(49), 103-125.

Oszlak, O. (1997). Estado y sociedad: ¿nuevas reglas de juego? Revista Del CLAD Reforma y Democracia, (9), 1-34.

Paura, V Zibecchi, C. (2014). Dinámicas institucionales, lógicas de los actores y territorio en el estudio de la política social. Veinte años de investigación en la Argentina. In L. Pautassi (Ed.), Marginaciones sociales en el área metropolitana de Buenos Aires. Acceso a la justicia, capacidades estatales y movilización legal. (pp. 333-396). Buenos Aires: Blblos. 
Perelmiter, L. (2012). Burocracia, pobreza y territorio. La política espacial de la asistencia en la Argentina reciente. In VII Jornadas de Sociología de la Universidad Nacional de General Sarmiento. Retrieved from https://www.ungs.edu.ar/wp-content/uploads/2012/11/Perelmiter-ponencia-versión-final.pdf

Rebón, J., \& Kasparian, D. (2015). La valoración social de las cooperativas en el área metropolitana de Buenos Aires. Una aproximación a partir de la investigación por encuesta. Revista Venezolana de Economía Social, (29), 11-37. Retrieved from https://www.academia.edu/35631082/La_valoración_social_ de_las_cooperativas_en_el_Área_Metropolitana_de_Buenos_Aires_Una_aproximación_a_partir_de_la_ investigación_por_encuesta

Repetto, F. (2010). Coordinación de Políticas Sociales: Abordaje Conceptual y Revisión de Experiencias Latinoamericanas. In Los desafíos de la coordinación y la integralidad de las políticas y gestión pública en América Latina (pp. 47-81). Buenos Aires: Jefatura de Gabinete de Ministros de la Nación.

Rofman, A. (2010). Introducción. En: Rofman, A. (Comps.) Sociedad y territorio en el conurbano bonaerense: un estudio de las condiciones socioeconómicas y sociopolíticas de cuatro partidos: San Miguel, José C. Paz, Moreno y Morón (pp.9-24). Los Polvorines: Universidad de Gral. Sarmiento.

Thwaites Rey, M (2001). Tecnócratas vs. Punteros: Nueva falacia de una vieja dicotomía: política vs. Administración. Revista Encrucijadas, №6, abril de 2001, pp1-12.

Wilson, W. (1980). El estudio de la administración. En Revista de Administración Pública Publicación Conmemorativa Del 25 Aniversario Del Instituto Nacional de Administración Pública, 275-294. 
